\title{
External validation of the Prostate Cancer Prevention Trial and the European Randomized Study of Screening for Prostate Cancer risk calculators in a Chinese cohort
}

\author{
Yao Zhu ${ }^{1,2, *}$, Jin-You Wang ${ }^{1,2, *}$, Yi-Jun Shen ${ }^{1,2}$, Bo Dai ${ }^{1,2}$, Chun-Guang Ma ${ }^{1,2}$, Wen-Jun Xiao ${ }^{1,2}$, Guo-Wen Lin ${ }^{1,2}$, \\ $\mathrm{Xu}$-Dong Yao ${ }^{1,2}$, Shi-Lin Zhang and Ding-Wei Ye $\mathrm{e}^{1,2}$
}

Several prediction models have been developed to estimate the outcomes of prostate biopsies. Most of these tools were designed for use with Western populations and have not been validated across different ethnic groups. Therefore, we evaluated the predictive value of the Prostate Cancer Prevention Trial (PCPT) and the European Randomized Study of Screening for Prostate Cancer (ERSPC) risk calculators in a Chinese cohort. Clinicopathological information was obtained from 495 Chinese men who had undergone extended prostate biopsies between January 2009 and March 2011. The estimated probabilities of prostate cancer and high-grade disease (Gleason $>6$ ) were calculated using the PCPT and ERSPC risk calculators. Overall measures, discrimination, calibration and clinical usefulness were assessed for the model evaluation. Of these patients, $28.7 \%$ were diagnosed with prostate cancer and $19.4 \%$ had high-grade disease. Compared to the PCPT model and the prostate-specific antigen (PSA) threshold of $4 \mathrm{ng} \mathrm{ml}^{-1}$, the ERSPC risk calculator exhibited better discriminative ability for predicting positive biopsies and high-grade disease (the area under the curve was 0.831 and 0.852 , respectively, $P<0.01$ for both). Decision curve analysis also suggested the favourable clinical utility of the ERSPC calculator in the validation dataset. Both prediction models demonstrated miscalibration: the risk of prostate cancer and high-grade disease was overestimated by approximately $20 \%$ for a wide range of predicted probabilities. In conclusion, the ERSPC risk calculator outperformed both the PCPT model and the PSA threshold of $4 \mathrm{ng} \mathrm{ml}^{-1}$ in predicting prostate cancer and high-grade disease in Chinese patients. However, the prediction tools derived from Western men significantly overestimated the probability of prostate cancer and high-grade disease compared to the outcomes of biopsies in a Chinese cohort.

Asian Journal of Andrology (2012) 14, 738-744; doi:10.1038/aja.2012.28; published online 7 May 2012

Keywords: European Randomized Study of Screening for Prostate Cancer (ERSPC); predictive value of tests; prostate cancer; prostatespecific antigen (PSA); Prostate Cancer Prevention Trial (PCPT)

\section{INTRODUCTION}

Prostate cancer is the second most frequently diagnosed cancer among men worldwide, with an estimated 914000 new cases in 2008. ${ }^{1}$ There are, however, striking geographical variations in the incidence rates of prostate cancer globally. For instance, the recorded rate (per 100000 ) of US blacks is 178.8 , which represents a 25 -fold increase compared to the rate of prostate cancer in Shanghai (6.9). ${ }^{1}$ One important reason for the large difference in prostate cancer incidence is the widespread use of prostate-specific antigen (PSA) testing in Western countries. ${ }^{2,3}$ In addition, accumulating evidence suggests that differences in lifestyle and genetic susceptibility are largely responsible for the observed regional/ethnic differences in prostate cancer risk. ${ }^{4}$

PSA has been commonly used by clinicians to estimate the probability of prostate cancer and to counsel patients regarding subsequent biopsies. However, the appropriate PSA threshold above which further evaluation with a biopsy of the prostate should be recommended to exclude prostate cancer remains controversial. ${ }^{5-7}$ PSA levels are a continuous parameter, so the higher the PSA value is, the higher the risk of prostate cancer. To overcome the drawbacks of PSA testing, several prediction models have been developed to assist in better risk classification. In 2006, investigators from the Prostate Cancer Prevention Trial (PCPT) reported a risk calculator based on age, race, PSA, digital rectal examination (DRE), family history and the history of a previous negative prostate biopsy. ${ }^{8}$ Similarly, the European Randomized Study of Screening for Prostate Cancer (ERSPC) section in Rotterdam constructed another risk calculator that includes PSA, DRE, transrectal ultrasound (TRUS) findings and prostate volume. ${ }^{9}$ The performance of these two risk calculators in Western populations has been assessed by external validation. ${ }^{10-13}$ However, the usefulness of these prediction tools in the Chinese population remains undefined. To evaluate this, we identified a cohort of patients who had undergone systematic prostate biopsies with a minimum of 10 cores at our

${ }^{1}$ Department of Urology, Fudan University, Shanghai Cancer Center, Shanghai 200032, China and ${ }^{2}$ Department of Oncology, Shanghai Medical College, Fudan University, Shanghai 200032, China

Correspondence: Dr DW Ye (dwye@shca.org.cn)

Received: 3 December 2011; Revised: 2 January 2012; Accepted: 19 February 2012; Published online: 7 May 2012

*These authors contributed equally to this work. 
institution. The predictive value of these two risk calculators was then externally validated and compared according to the standard framework. ${ }^{14}$

\section{MATERIALS AND METHODS}

\section{Patient population and risk calculations}

We retrospectively reviewed the medical records of patients who had undergone TRUS-guided prostate biopsies at Fudan University Shanghai Cancer Center between January 2009 and March 2011. The indications of prostate biopsy were a PSA level greater than $4 \mathrm{ng} \mathrm{ml}^{-1}$ and/or suspicious results from a DRE or a TRUS. Of 609 assessable patients, 114 were excluded because of extreme variables (PSA level $>50 \mathrm{ng} \mathrm{ml}^{-1}$ or prostate volume $>150 \mathrm{ml}$ ) or rare types of prostate cancer. Therefore, a total of 495 cases, including 402 initial biopsies and 93 repeat biopsies, were available for further analysis. Initial biopsies and repeat biopsies were performed according to the institution's protocol with a minimum of 10 and 12 cores, respectively. All specimens were independently evaluated by two genitourinary pathologists for diagnoses and Gleason scores.

Two formulas from PCPT to predict the probability of prostate cancer and high-grade disease (Gleason $>6$ ) were obtained from the authors' web site. ${ }^{8}$ Individual risk estimations using the ERSPC model were calculated using online calculators 3 and 5 for patients undergoing initial biopsies and repeat biopsies, respectively. ${ }^{9}$ Variables were coded and transformed according to the requirements of the ERSPC and PCPT risk calculators. To better define the value of the risk calculators, PSA levels were used as a reference prediction tool in the analyses.

This study was approved by the institutional review board, and written inform consent was obtained from each patient.

\section{Statistical analyses}

Descriptive statistics of the cohort were reported and compared to those of the datasets used to develop the risk calculators. Multivariate logistic regression analyses were carried out to assess the predictive variables. For high-grade disease, a multinomial model was applied with three potential outcomes-no cancer, low-grade cancer and high-grade cancer. The performance of each risk calculator was evaluated statistically on the basis of its discrimination, calibration and clinical usefulness. ${ }^{14}$ The area under the receiver operating characteristic (ROC) curve was used to compare the ability to discriminate between patients with prostate cancer from those without prostate cancer. The comparisons of the areas under the curve (AUCs) were made using the method of DeLong et al. ${ }^{15}$ Calibration refers to a measure of the level of agreement between the observed outcomes in the validation cohort $(n=495)$ and the predicted probabilities calculated using the risk calculators. The extent of over/underestimation comparing the observed rates to the predicted rates was explored graphically using calibration plots. Calibration-in-the-large was evaluated by fitting a logistic regression model with the predictions of the model as an offset variable. The intercept, which ideally should be zero, indicates whether the predictions were systematically too low or too high. The calibration slope reflects the average effects of the predictors in the model and was estimated in a logistic regression model with the logit of the model predictions as the only predictor. For a perfect model, the slope would be equal to 1 . Clinical utility was assessed using decision curve analyses. ${ }^{16}$ These analyses estimate the 'net benefit' of prediction models by summing the benefits (true-positive biopsies) and subtracting the negative outcomes (false-positive biopsies). The latter are weighted by a factor related to the relative harm of a missed cancer diagnosis versus an unnecessary biopsy. The weighting is derived from the threshold probability of prostate cancer at which a patient would opt for a biopsy, which can vary from patient to patient. When interpreting decision curves, the model with the highest net benefit at a particular threshold probability should be chosen. For all analyses, two-sided $P$ values $<0.05$ were considered to be statistically significant. Statistical analyses were performed using R 2.13.0 software (R Foundation for Statistical Computing, Vienna, Austria). ${ }^{17}$

\section{RESULTS}

Table 1 shows the clinicopathological characteristics of the participants enrolled in the current study and those from the ERSPC and PCPT datasets ${ }^{8,9}$ (Roobol MJ, pers. commun.). The age distributions were similar among the three patient cohorts. The study population from our institution, however, had the highest median PSA levels. The differences in baseline characteristics were the most obvious between patients from our cohort and those from the PCPT study. Men who were referred to our centre seldom had a family history of prostate cancer but had a high probability of abnormal DRE findings.

Multivariate logistic regression analyses of predictors were performed for biopsy outcomes (Table 2). Because of its extremely low prevalence $(0.6 \%)$, family history was not included as a prognostic factor in this cohort. Statistical analyses revealed that age, PSA levels, prostate volume and abnormal DREs were significant prognostic factors for prostate cancer and high-grade disease.

External validations of the ERSPC and PCPT risk calculators were performed according to a recently described framework. ${ }^{14}$ First, the detailed performance statistics of each prediction model were assessed (Table 3). Second, the ROC curves of the risk calculators and the $4 \mathrm{ng} \mathrm{ml}^{-1}$ PSA threshold were compared. Various cut-offs and their corresponding test sensitivities and specificities were listed for the convenience of clinical practice. Third, calibration plots were drawn to demonstrate the discrepancies between the predicted and the observed probabilities over from $0 \%$ to $100 \%$. Finally, the clinical utility of the prediction models was assessed using decision curve analysis. The net benefit and net reduction of each model at various thresholds was calculated by subtracting that of the traditional PSA cut-off.

In this cohort, the AUC for PSA to discriminate any prostate cancer and high-grade disease were $0.75(0.70-0.80)$ and $0.75(0.69-0.81)$, respectively. For estimating the probability of a positive biopsy, the ERSPC risk calculator exhibited a higher AUC than either the PCPT model or the PSA threshold of $4 \mathrm{ng} \mathrm{ml}^{-1}$ (both $P<0.01$, as shown in Figure 1a). However, the calibration plots of the two models were far from ideal (Figure $\mathbf{2} \mathbf{a}$ and $\mathbf{b}$ ). The risk of malignant disease was overestimated by more than $20 \%$ for predicted probabilities between $40 \%$ and $80 \%$. The decision curve analysis indicated that the ERSPC model outperformed both the PCPT model and the PSA criterion (Figure 3a and b). Similarly, the ERSPC model also showed better discriminative ability (both $P<0.01$, as shown in Figure $\mathbf{1 b}$ ) and clinical usefulness (Figure $3 \mathbf{c}$ and $\mathbf{d}$ ) than both the PCPT model and the PSA criterion in predicting high-grade cancer. However, the predicted probabilities provided by the two models were approximately $20 \%$ higher than the observed frequency in a wide range of patients (Figure $2 \mathrm{c}$ and $\mathbf{d}$ ).

\section{DISCUSSION}

By incorporating several predictors, the ERSPC and PCPT risk calculators were developed to facilitate making appropriate decisions regarding prostate biopsies. ${ }^{8,9}$ The two prediction models have been previously applied to Western populations, ${ }^{10-13}$ but their performance in Chinese men had been unknown. The objective of this study 
Table 1 Descriptive data of the study cohort and the datasets used to develop the ERSPC and PCPT risk calculators

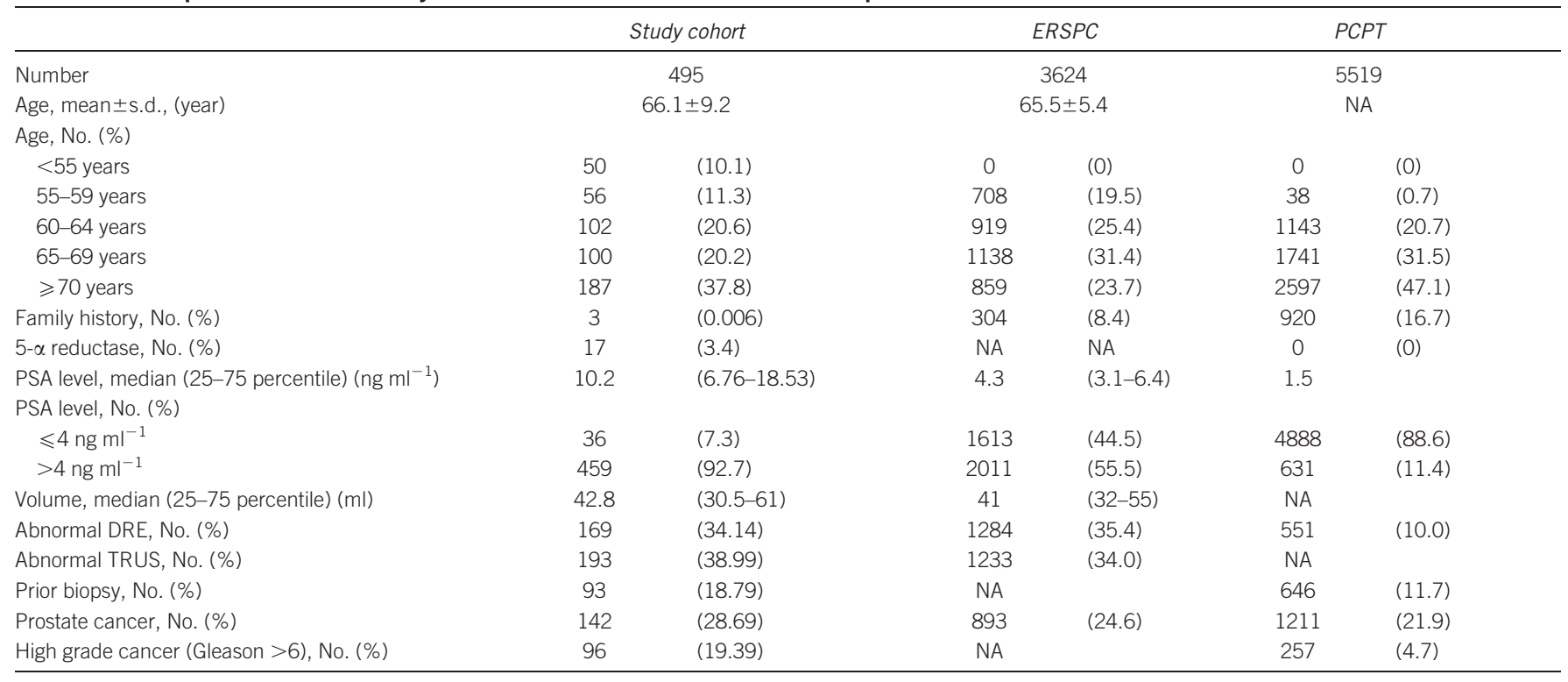

Abbreviations: DRE, digital rectal examination; ERSPC, European Randomized Study of Screening for Prostate Cancer; NA, not available; PCPT, Prostate Cancer Prevention Trial; PSA, prostate-specific antigen; TRUS, transrectal ultrasound.

was to externally validate the ERSPC and PCPT risk calculators in a Chinese cohort referred to a regional cancer centre. For predicting the risk of positive biopsy, the ERSPC model showed better discriminative ability and clinical utility than the PCPT risk calculator and the use of PSA levels alone. However, the prediction tool strongly overestimated the probability of prostate cancer in the study cohort. Similar findings were observed after applying the two models for estimating the probability of high-grade disease.

Ethnic variation is an important determinant of the incidence and characteristics of prostate cancer. ${ }^{4}$ The standardized incidence (per 100000 individuals) of prostate cancer reported between 2003 and 2007 was 230 for African American men and 81 in Asian American and Pacific Islander males. ${ }^{18}$ Even among Asian Americans, the incidence of prostate cancer varies greatly, with the highest rate observed in Filipinos and the lowest in Koreans. ${ }^{19}$ Although PSA screening markedly influences the incidence of prostate cancer, it cannot account for all of the discrepancies between different ethnical groups. We found the frequency of prostate cancer in radical cystoprostatectomy specimens to be only $3.3 \%$ (3/92) in Chinese men, which is much lower than that reported in Western countries. ${ }^{20} \mathrm{~A}$ recent study provided further evidence about distinctive genomic alterations between

Table 2 Multivariate logistic regression analysis of predictors of biopsy outcomes

\begin{tabular}{lcccccccc}
\hline & \multicolumn{3}{c}{ Prostate cancer } & & \multicolumn{3}{c}{ High-grade disease } \\
\cline { 2 - 3 } Variables & $\begin{array}{c}\text { Odds } \\
\text { ratio }\end{array}$ & $95 \% \mathrm{Cl}$ & P value & & $\begin{array}{c}\text { Odds } \\
\text { ratio }\end{array}$ & $95 \% \mathrm{Cl}$ & P value \\
& & & & & & \\
\hline Ln(age) & 1.91 & $1.34-2.72$ & 0.0003 & & 1.73 & $1.25-2.40$ & 0.001 \\
Ln(PSA) & 4.09 & $2.77-6.04$ & $<0.0001$ & & 3.82 & $2.66-5.48$ & $<0.0001$ \\
Ln(volume) & 0.17 & $0.11-0.27$ & $<0.0001$ & & 0.21 & $0.14-0.32$ & $<0.0001$ \\
DRE & 3.55 & $2.05-6.16$ & $<0.0001$ & & 3.75 & $2.25-6.24$ & $<0.0001$ \\
TRUS & 1.41 & $0.82-2.42$ & 0.217 & & 1.33 & $0.80-2.20$ & 0.277 \\
Prior biopsy & 0.99 & $0.52-1.92$ & 0.986 & & 0.79 & $0.43-1.45$ & 0.451 \\
\hline
\end{tabular}

Abbreviations: $\mathrm{Cl}$, confidence interval; DRE, digital rectal examination; Ln, natural logarithm; PSA, prostate-specific antigen; TRUS, transrectal ultrasound. prostate cancers in individuals from Chinese men and men from Western populations. Certain somatic genomic changes that are commonly found in Western cases are only rarely observed in Chinese patients. ${ }^{21}$ Different carcinogenic mechanisms may subsequently affect prostate cancer disease characteristics and warrant the ethnicbased management of prostate cancer.

Recently, Yoon et al. ${ }^{22}$ validated the ERSPC risk calculator 3 in 602 Korean patients. The observed AUC was 0.88 (95\% confidence interval (CI): 0.86-0.90), but the calibration plot showed remarkable miscalibration: the predicted probabilities were nearly $20 \%$ higher than the observed frequency for predictions from $30 \%$ to $90 \%{ }^{22}$ Similar to these previous findings, we found that the prediction models derived from Western patients overrated the probability of prostate cancer and high-grade disease by approximately $20 \%$. Because we used an extended 10 core biopsy in all patients, the observed overestimation of risk is unlikely to be due to missed cancer diagnoses by biopsies. Several reasons may account for the low probability of prostate cancer in this study cohort. First, although age, PSA level, prostate volume and DRE were identified as independent prognostic factors in both the validation and development sets, the strength of these associations may vary in different settings. In the ERSPC study, the effect of PSA levels on biopsy outcomes was greater under the $3 \mathrm{ng} \mathrm{ml}^{-1}$ threshold than at PSA levels above $3 \mathrm{ng} \mathrm{ml}{ }^{-1} \cdot{ }^{23}$ Furthermore, both TRUS and DRE significantly increased the detection rate of prostate cancer in subjects with PSA levels below $4 \mathrm{ng} \mathrm{ml}^{-1} \cdot{ }^{24,25}$ In our series, multivariate analysis demonstrated that the odds ratios of PSA and prostate volume were 4.09 and 0.17 , respectively. In contrast, the effect measures of PSA and prostate volume in the ERSPC study were 42.8 and 0.02 , respectively. ${ }^{23}$ Therefore, the risk calculators derived from screening datasets may overestimate the effects of prognostic factors when applied to men with elevated PSA levels. Second, the diagnostic characteristics of the assessed predictors exhibited significant variation between different ethnic groups. Using a PSA level of $4.1 \mathrm{ng} \mathrm{ml}^{-1}$ as the threshold for biopsy, the sensitivity and the specificity of the test were $35 \%$ and $88 \%$, respectively, in men over 60 years of age from a North American cohort. ${ }^{5}$ However, similar criteria resulted 
Table 3 Assessments of the performance of the ERSPC and PCPT risk calculators in Chinese patients $(n=495)$

\begin{tabular}{|c|c|c|c|c|}
\hline \multirow{2}{*}{ Performance measures } & \multicolumn{2}{|c|}{ Prostate cancer } & \multicolumn{2}{|c|}{ High-grade disease } \\
\hline & ERSPC & $P C P T$ & ERSPC & $P C P T$ \\
\hline \multicolumn{5}{|l|}{ Overall } \\
\hline \multicolumn{5}{|l|}{ Discrimination } \\
\hline $\mathrm{A} \cup \mathrm{C}(95 \% \mathrm{Cl})$ & $0.831(0.790-0.872)$ & $0.783(0.737-0.830)$ & $0.852(0.807-0.897)$ & $0.813(0.764-0.862)$ \\
\hline Discrimination slope & 0.294 & 0.111 & 0.433 & 0.280 \\
\hline Observed outcome (\%) & 29 & 29 & 19 & 19 \\
\hline Calibration-in-the-large $(95 \% \mathrm{Cl})$ & $-1.764(-2.073,-1.455)$ & $-1.107(-1.353,-0.860)$ & $-1.145(-1.412,-0.877)$ & $-0.939(-1.195,-0.683)$ \\
\hline Calibration slope $(95 \% \mathrm{Cl})$ & $1.402(1.104,1.700)$ & $0.873(0.707,1.039)$ & $0.748(0.596,0.901)$ & $1.051(0.818,1.283)$ \\
\hline Hosmer-Lemeshow test & $\chi^{2}=227.047, P<0.001$ & $\chi^{2}=108.517, P<0.001$ & $\chi^{2}=105.403, P<0.001$ & $\chi^{2}=60.413, P<0.001$ \\
\hline
\end{tabular}

Abbreviations: AUC, area under the curve; Cl, confidence interval; ERSPC, European Randomized Study of Screening for Prostate Cancer; PCPT, Prostate Cancer Prevention Trial.

in a sensitivity of $96 \%$ and a specificity of $14 \%$ in a cohort of 1236 Chinese patients. ${ }^{6}$ From a mass screening study of Chinese individuals, Gao et al. ${ }^{26}$ reported that the detection rate of prostate cancer was $14.8 \%$ in the PSA range of $4-10 \mathrm{ng} \mathrm{ml}^{-1}$ and $29 \%$ in the PSA range of $10-20 \mathrm{ng} \mathrm{ml}^{-1}$. In contrast, similar criteria yielded an approximately twofold increase in the probability of prostate cancer in Americans. ${ }^{27}$ A similar phenomenon was observed for abnormal DRE results: $52 \%$ of white, $58 \%$ of black and $34 \%$ of Asian/Pacific Islander men had a positive biopsy, which corresponds to an approximately 40\% lower rate in Asians/Pacific Islanders when compared to white or black men. ${ }^{28}$ The difference was more prominent in men with PSA levels less than $10 \mathrm{ng} \mathrm{ml}^{-1}$ and abnormal DRE findings. Indeed, in these individuals, the rate of prostate cancer diagnosis was $42.8 \%$,
$52.0 \%$ and $13.5 \%$ for white, black and Asian/Pacific Islander men, respectively. ${ }^{28}$ Finally, the prognostic significance of PSA levels and DRE results may be limited in Chinese patients. Because PSA screenings are largely unavailable in China, many referral patients may have a history of chronic inflammation or benign prostate hyperplasia that subsequently results in elevated PSA levels and potentially contributes to abnormal DRE results.

Several studies have reported conflicting results. For example, Ito et al. ${ }^{29}$ had reported the probability of prostate cancer in a Japanese screening cohort that was observed over a 4 -year period. Only men between the ages of 55 and 74 who had PSA levels less than $4 \mathrm{ng} \mathrm{ml}^{-1}$ and did not exhibit abnormal DRE results were initially enrolled. Interestingly, Ito et al. ${ }^{29}$ found a similar risk of developing prostate cancer as that reported in

\section{a}

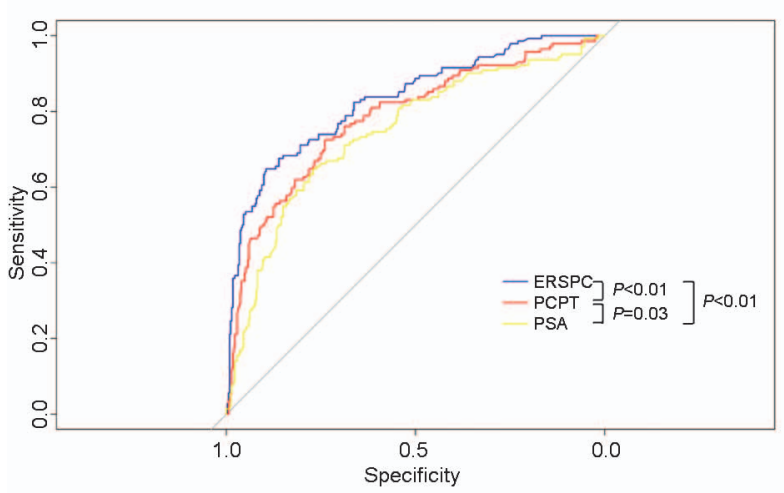

b

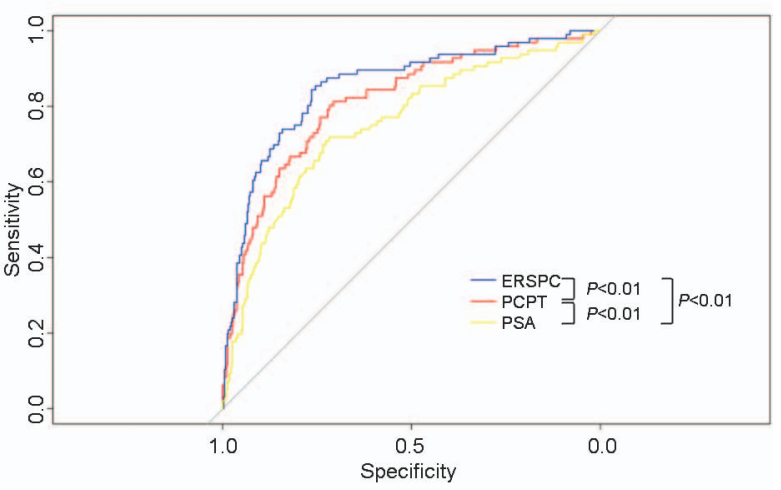

\begin{tabular}{|c|c|c|c|c|c|c|c|c|}
\hline \multirow{3}{*}{ Cutoff } & \multicolumn{4}{|c|}{ Prostate cancer } & \multicolumn{4}{|c|}{ High-grade disease } \\
\hline & \multicolumn{2}{|c|}{ ERSPC } & \multicolumn{2}{|c|}{ PCPT } & \multicolumn{2}{|c|}{ ERSPC } & \multicolumn{2}{|c|}{ PCPT } \\
\hline & Sensitivity & Specificity & Sensitivity & Specificity & Sensitivity & Specificity & Sensitivity & Specificity \\
\hline $10 \%$ & $100 \%$ & $13.6 \%$ & $100 \%$ & $0.57 \%$ & $93.8 \%$ & $42.1 \%$ & $97.9 \%$ & $14.3 \%$ \\
\hline $20 \%$ & $94.4 \%$ & $33.4 \%$ & $99.3 \%$ & $2.3 \%$ & $89.6 \%$ & $61.7 \%$ & $91.7 \%$ & $46.6 \%$ \\
\hline $30 \%$ & $89.4 \%$ & $49.0 \%$ & $98.6 \%$ & $5.9 \%$ & $86.5 \%$ & $73.7 \%$ & $82.3 \%$ & $65.2 \%$ \\
\hline $40 \%$ & $83.1 \%$ & $64.3 \%$ & $96.5 \%$ & $15.9 \%$ & $78.1 \%$ & $78.7 \%$ & $70.8 \%$ & $76 . \%$ \\
\hline
\end{tabular}

Figure 1 ROC curves of the risk calculators and the PSA threshold of $4 \mathrm{ng} \mathrm{ml^{-1 }}$ for prostate cancer (a) and high-grade disease (b) in the validation cohort ( $n=495$ ). Pairwise comparisons of ROC curves are shown in the bottom-right corner of the figure. The table displays the sensitivity and specificity of each risk calculators at different cut-offs. For example, using the ERSPC risk calculator in this cohort, a cut-off of $30 \%$ corresponds to a sensitivity of $89.4 \%$ and a specificity of $49.0 \%$ in detecting prostate cancer. ERSPC, European Randomized Study of Screening for Prostate Cancer; PSA, prostate-specific antigen; ROC, receiver operating characteristic. 

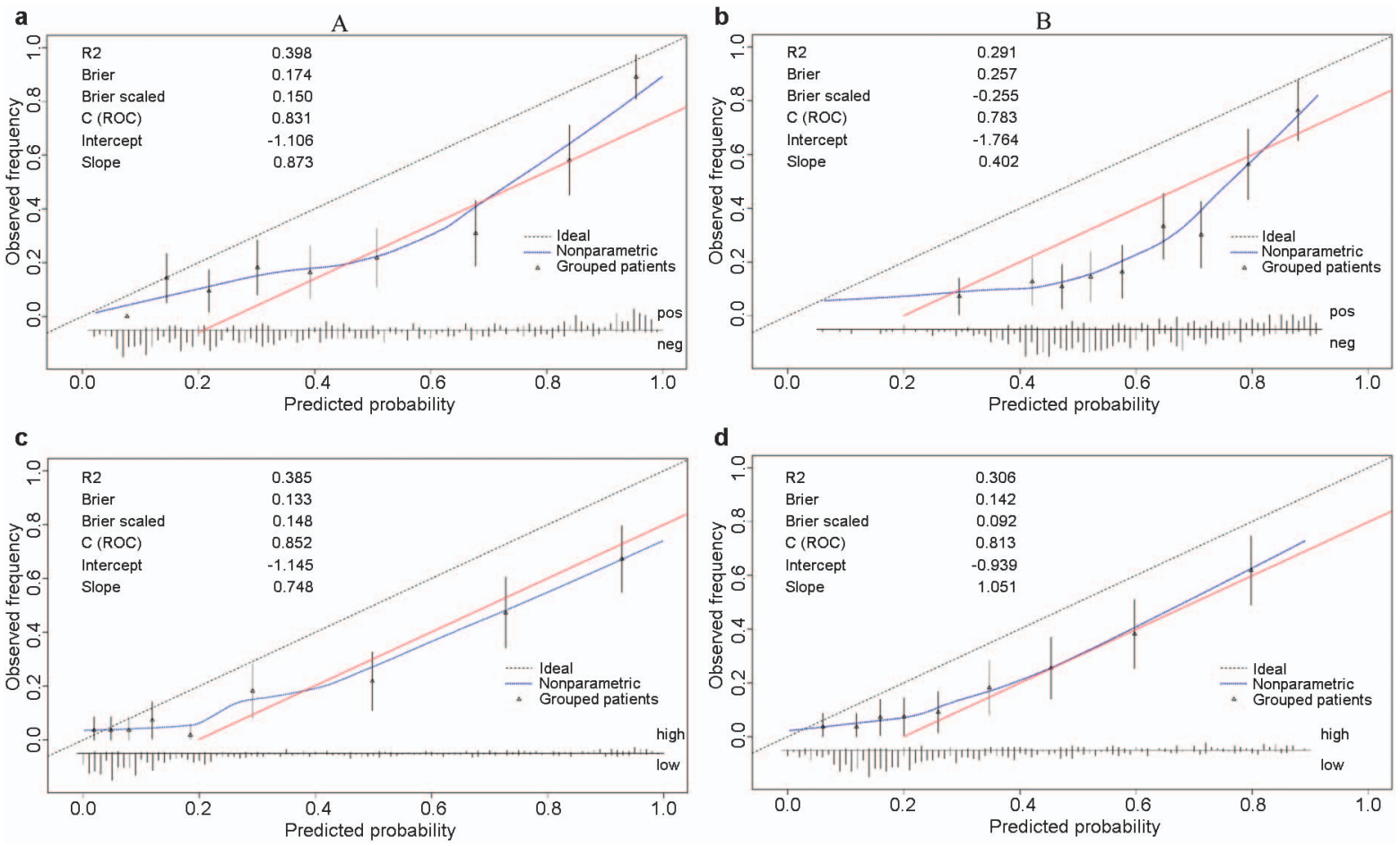

Figure 2 Calibration plots of the risk calculators in the validation cohort ( $n=495)$ : ERSPC (a) and PCPT (b) risk calculators for prostate cancer; ERSPC (c) and PCPT (d) risk calculator for high-grade disease. The $45^{\circ}$ dashed line represents an ideal prediction, in which the predicted and actual probabilities are identical. The actual performance of each risk calculator is represented by the blue dotted line. The red solid line indicates that the predicted risk was $20 \%$ higher than the observed probability. The spike histogram at the bottom of the figure shows the distribution of the individual predicted probabilities. ERSPC, European Randomized Study of Screening for Prostate Cancer; PCPT, Prostate Cancer Prevention Trial; ROC, receiver operating characteristic.

the ERSPC study after adjusting for initial PSA levels. However, because of the relatively short follow-up period (4 years) of Ito et al. 's study, it is likely that their results actually reflect the probability of active prostate cancers becoming clinically detectable. As revealed by autopsy studies, the actual prevalence of prostate cancer is strikingly lower in Asian areas than in other parts of the world. ${ }^{30,31}$ Furthermore, the characteristics of the enrolled subjects varied between the Japanese and the Rotterdam cohort: $49.8 \%$ of the men in the Japanese study had initial PSA levels below $1 \mathrm{ng} \mathrm{ml}^{-1}$, compared to only $25.9 \%$ for individuals from the Rotterdam cohort. ${ }^{29}$ It is therefore difficult to observe significant differences between these relatively low-risk groups given the relatively short follow-up period.

Our results indicate that the ERSPC risk calculator exhibits superior discrimination and calibration than the PCPT model. One potential explanation for this observation is that the ERSPC model includes more predictive prognostic factors, such as prostate volume and TRUS findings. In contrast, the inclusion of family history did not enhance the predictive ability of the PCPT risk calculator, because only three patients from our cohort had a positive family history. This finding is similar to that from another population-based study in Shanghai, in which none of the 238 subjects reported a family history of prostate cancer. ${ }^{32}$ Furthermore, the study population used to develop the PCPT risk calculator was quite different from our cohort. For instance, the percentage of men with PSA levels above $4 \mathrm{ng} \mathrm{ml}^{-1}$ was $92.7 \%$ in our study but only $11.4 \%$ in the PCPT cohort. Therefore, the observed lack of predictive ability may be largely due to the significant differences in patient characteristics between the population used to develop the PCPT risk calculator and the current cohort.
Our study had several limitations. First, because the variables and outcomes were retrospectively collected from a tertiary cancer centre, the results may have been influenced by the heterogeneity of patients, tumours and biopsy techniques. Second, most (92.7\%) of the individuals enrolled in this study exhibited PSA values greater than $4 \mathrm{ng} \mathrm{ml}^{-1}$. Thus, the performance of these risk calculators in Chinese men underwent PSA screen remains to be determined. Furthermore, the long-term outcomes of these risk calculators in Chinese prostate cancer patients should be determined because of possible distinctive disease characteristics observed in this population. Nevertheless, our study may provide a key step towards understanding the optimal prostate cancer management strategies in different ethnic groups.

In conclusion, compared to the PCPT risk calculator, the ERSPC model exhibited better discriminative ability for prostate cancer and high-grade disease in Chinese patients. However, the two prediction tools significantly overestimated the probability of biopsy outcome by approximately $20 \%$. The underlying variations in carcinogenesis pathways between different ethnic groups require further investigation.

\section{AUTHOR CONTRIBUTIONS}

YZ, JYW and DWY conceived and designed the study. YJS, BD, CGM, WJX, GWL, XDY and SLZ collected the data and performed the studies. YZ and JYW performed the statistical analyses. YZ, JYW and DWY wrote the manuscript with input from all co-authors. All authors revised the manuscript for intellectual content and approved the final version.

\section{COMPETING FINANCIAL INTERESTS}

The authors declare no competing financial interests. 


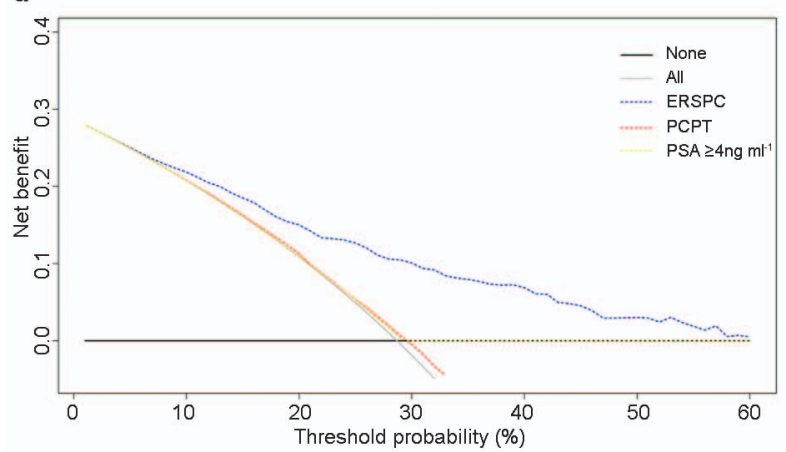

C

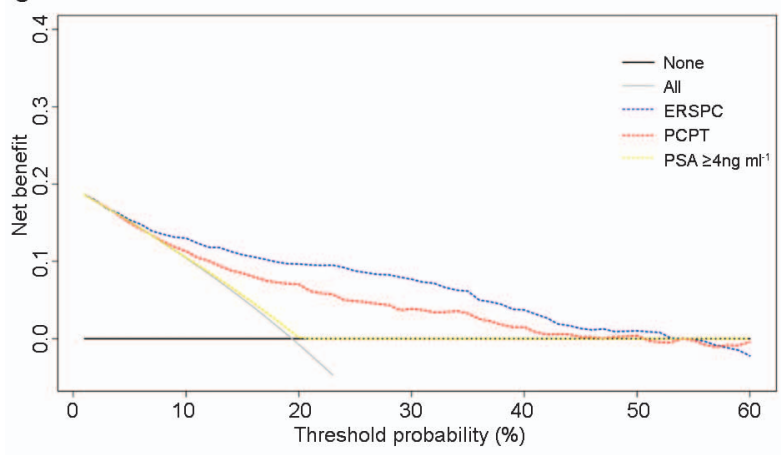

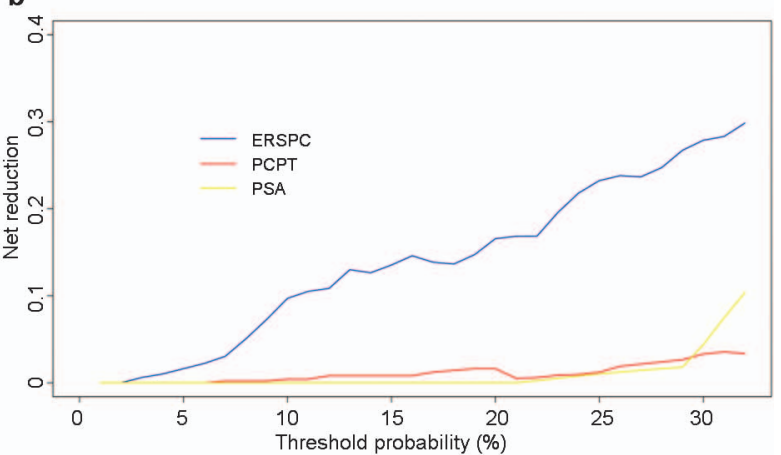

d

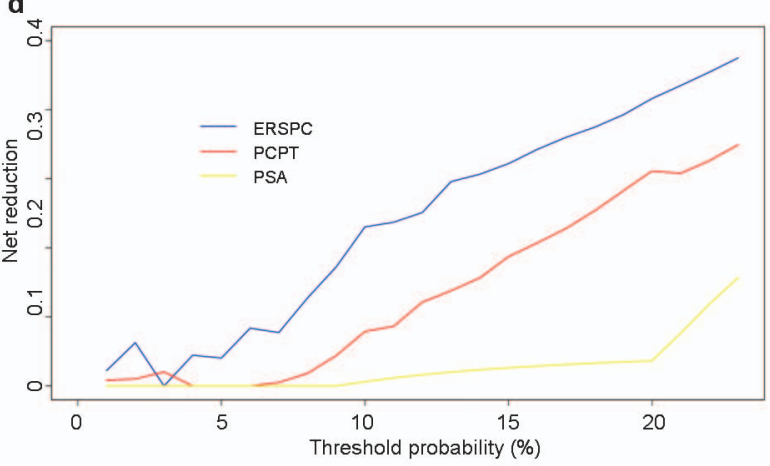

\begin{tabular}{|c|c|c|c|c|c|c|c|c|}
\hline \multirow{3}{*}{$\begin{array}{l}\text { Threshold } \\
\text { probability (\%) }\end{array}$} & \multicolumn{4}{|c|}{ Prostate cancer } & \multicolumn{4}{|c|}{ High-grade disease } \\
\hline & \multicolumn{2}{|c|}{ ERSPC } & \multicolumn{2}{|c|}{ PCPT } & \multicolumn{2}{|c|}{ ERSPC } & \multicolumn{2}{|c|}{ PCPT } \\
\hline & Net benefit (\%) & Net reduction (\%) & Net benefit (\%) & Net reduction (\%) & Net benefit (\%) & Net reduction (\%) & Net benefit (\%) & Net reduction (\%) \\
\hline $10 \%$ & 1.1 & 9.7 & 0 & 0.4 & 2.5 & 22.4 & 0.8 & 7.3 \\
\hline $20 \%$ & 4.1 & 16.6 & 0.4 & 1.6 & 9.5 & 38.0 & 6.9 & 27.5 \\
\hline $30 \%$ & 10.1 & 23.5 & -0.5 & 0 & 7.7 & 18.0 & 3.8 & 9.0 \\
\hline $40 \%$ & 6.9 & 10.3 & NA & NA & 3.7 & 5.6 & 1.5 & 2.2 \\
\hline
\end{tabular}

Net benefit and reduction for each of the risk calculators were calculated compared with the strategy based on PSA alone (biopsy when PSA $\geq 4 \mathrm{ng} \mathrm{ml}^{-1}$ )

Figure 3 Decision curve analyses of the risk calculators and the PSA threshold of $4 \mathrm{ng} \mathrm{ml}^{-1}$ in the validation cohort ( $n=495$ ): net benefit (a) and reduction (b) curves for prostate cancer; net benefit (c) and reduction (d) curves for high-grade disease. The table displays the net benefit and reduction statistics at different threshold probabilities. For example, using the ERSPC risk calculator in this cohort, a threshold probability of $30 \%$ corresponds to a net benefit of $10.1 \%$ and a net reduction of $23.5 \%$ over the PSA cut-off of $4 \mathrm{ng} \mathrm{ml}^{-1}$ in detecting prostate cancer. ERSPC, European Randomized Study of Screening for Prostate Cancer; PCPT, Prostate Cancer Prevention Trial; PSA, prostate-specific antigen.

\section{ACKNOWLEDGMENTS}

This study was supported by the Shanghai Outstanding Leader Program in Academic Discipline (No. 09XD1401200) and the National Natural Science Foundation of China (No. 30801149). The authors would like to thank Ewout W Steyerberg and Monique J. Roobol from Erasmus Medical Center for their kind help in preparing the manuscript and their thoughtful suggestions.

1 Ferlay J, Shin HR, Bray F, Forman D, Mathers C et al. GLOBOCAN 2008 v1.2, Cancer Incidence and Mortality Worldwide: IARC CancerBase No. 10. Lyon: International Agency for Research on Cancer; 2010. http://globocan.iarc.fr (accessed on 11 August 2011).

2 Etzioni R, Penson DF, Legler JM, di Tommaso D, Boer R et al. Overdiagnosis due to prostate-specific antigen screening: lessons from U.S. prostate cancer incidence trends. J Natl Cancer Inst 2002; 94: 981-90.

3 Zhang L, Yang BX, Zhang HT, Wang JG, Wang HL et al. Prostate cancer: an emerging threat to the health of aging men in Asia. Asian J Androl 2011; 13: 574-8.

4 Freedland SJ, Isaacs WB. Explaining racial differences in prostate cancer in the United States: sociology or biology? Prostate 2005; 62: 243-52.

5 Punglia RS, D'Amico AV, Catalona WJ, Roehl KA, Kuntz KM. Effect of verification bias on screening for prostate cancer by measurement of prostate-specific antigen. $N$ Engl J Med 2003; 349: 335-42.

6 Wu TT, Huang JK. The clinical usefulness of prostate-specific antigen (PSA) level and age-specific PSA reference ranges for detecting prostate cancer in Chinese. Urol Int 2004; 72: 208-11.
7 Phillips JM, Crawford ED. Prostate-specific antigen (PSA) screening: has the pendulum swung too far? Asian J Androl 2011; 13: 655-6.

8 Thompson IM, Ankerst DP, Chi C, Goodman PJ, Tangen CM et al. Assessing prostate cancer risk: results from the Prostate Cancer Prevention Trial. J Natl Cancer Inst 2006; 98: 529-34.

9 Roobol MJ, Steyerberg EW, Kranse R, Wolters T, van den Bergh RC et al. A risk-based strategy improves prostate-specific antigen-driven detection of prostate cancer. Eur Urol 2010; 57: 79-85.

10 Parekh DJ, Ankerst DP, Higgins BA, Hernandez J, Canby-Hagino E et al. External validation of the Prostate Cancer Prevention Trial risk calculator in a screened population. Urology 2006; 68: 1152-5.

11 Eyre SJ, Ankerst DP, Wei JT, Nair PV, Regan MM et al. Validation in a multiple urology practice cohort of the Prostate Cancer Prevention Trial calculator for predicting prostate cancer detection. J Urol 2009; 182: 2653-8.

12 Cavadas V, Osorio L, Sabell F, Teves F, Branco F et al. Prostate cancer prevention trial and European randomized study of screening for prostate cancer risk calculators: a performance comparison in a contemporary screened cohort. Eur Urol 2010; 58: 551-8.

13 Nguyen CT, Yu C, Moussa A, Kattan MW, Jones JS. Performance of prostate cancer prevention trial risk calculator in a contemporary cohort screened for prostate cancer and diagnosed by extended prostate biopsy. J Urol 2010; 183: 529-33.

14 Steyerberg EW, Vickers AJ, Cook NR, Gerds T, Gonen M et al. Assessing the performance of prediction models: a framework for traditional and novel measures. Epidemiology 2010; 21: 128-38.

15 DeLong ER, DeLong DM, Clarke-Pearson DL. Comparing the areas under two or more correlated receiver operating characteristic curves: a nonparametric approach. Biometrics 1988; 44: 837-45.

16 Vickers AJ, Elkin EB. Decision curve analysis: a novel method for evaluating prediction models. Med Decis Making 2006; 26: 565-74. 
neg

Validation of prostate cancer risk calculators in Chinese

Y Zhu et al

744

17 R Development Core Team. R: A Language and Environment for Statistical Computing. Vienna: R Foundation for Statistical Computing; 2011. http://www.R-project.org/

18 Siege R, Ward E, Brawley 0, Jamal A. Cancer statistics, 2011: the impact of eliminating socioeconomic and racial disparities on premature cancer deaths. $C A$ Cancer J Clii 2011; 61: 212-36.

19 McCracken M, Olsen M, Chen MS Jr, Jemal A, Thun M et al. Cancer incidence, mortality, and associated risk factors among Asian Americans of Chinese, Filipino, Vietnamese, Korean, and Japanese ethnicities. CA Cancer J Chin 2007; 57: 190205.

20 Zhu YP, Ye DW, Yo XD, Zhang SL, Dali B et al. Prevalence of incidental prostate cancer in patients undergoing radical cystoprostatectomy: data from China and other Asian countries. Asian J Androl 2009; 11: 104-8.

21 Mao X, Mu Y, Boyd LK, Pen G, Lin D et al. Distinct genomic alterations in prostate cancers in Chinese and Western populations suggest alternative pathways of prostate carcinogenesis. Cancer Res 2010; 70: 5207-12.

22 Poon DK, Park JY, Mon S, Park MS, Moon DG et al. Can the prostate risk calculator based on western population be applied to Asian population? The Prostate; e-pub ahead of print 11 August 2011; doi:10.1002/pros.21475.

23 van Vugt HA, Roobol MJ, Kranse R, Maattanen L, Finne P et al. Prediction of prostate cancer in unscreened men: external validation of a risk calculator. Eur J Cancer 2011; 47: 903-9.

24 Yamamoto T, Ito K, Obi M, Kubota Y, Suzuki K et al. Diagnostic significance of digital rectal examination and transrectal ultrasonography in men with prostate-specific antigen levels of $4 \mathrm{ng} / \mathrm{ml}$ or less. Urology 2001; 58: 994-8.
25 Fowler JJ, Bigler SA, Farabaugh PB, Wilson SS. Prostate cancer detection in Black and White men with abnormal digital rectal examination and prostate specific antigen less then $4 \mathrm{ng} / \mathrm{ml}$. J Ural 2000; 164: 1961-3.

26 Gao HW, Li YL, Wu S, Wang YS, Chang HF et al. Mass screening of prostate cancer in a Chinese population: the relationship between pathological features of prostate cancer and serum prostate specific antigen. Asian J Androl 2005; 7: 159-63.

27 Catalonia WJ, Richie JP, Ahmann FR, Hudson MA, Scardino PT et al. Comparison of digital rectal examination and serum prostate specific antigen in the early detection of prostate cancer: results of a multicenter clinical trial of 6,630 men. J Ural 1994; 151. 1283-90.

28 Elliott CS, Shinghal R, Presti JC Jr. Racial variations in the performance of prostate specific antigen and prostate specific antigen density in the era of extended prostate biopsy schemes. J Ural 2008; 180: 1318-23.

29 Ito K, Raaijmakers R, Roobol M, Wildhagen M, Yamanaka H et al. Prostate carcinoma detection and increased prostate-specific antigen levels after 4 years in Dutch and Japanese males who had no evidence of disease at initial screening. Cancer 2005; 103: 242-50.

30 Yatani R, Chigusa I, Akazaki K, Stemmermann GN, Welsh RA et al. Geographic pathology of latent prostatic carcinoma. Int J Cancer 1982; 29: 611-6.

31 Breslow N, Chan CW, Dhow G, Drury RA, Franks LM et al. Latent carcinoma of prostate at autopsy in seven areas. The International Agency for Research on Cancer, Lyons, France. Int J Cancer 1977; 20: 680-8.

32 Bail Y, Gao YT, Deng J, Sesterhenn IA, Fraumeni JF et al. Risk of prostate cancer and family history of cancer: a population-based study in China. Prostate Cancer Prostatic Dis 2005; 8: 60-5.

Asian Journal of Andrology 